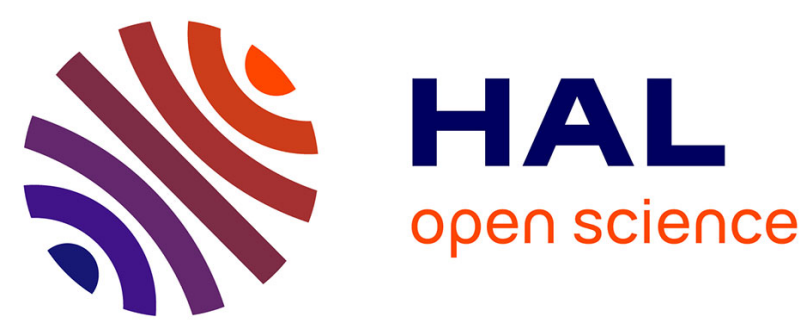

\title{
Tracking the Algebraic Multiplicity of Crossing Imaginary Roots for Generic Quasipolynomials: A Vandermonde-Based Approach
}

Islam Boussaada, Silviu-Iulian Niculescu

\section{- To cite this version:}

Islam Boussaada, Silviu-Iulian Niculescu. Tracking the Algebraic Multiplicity of Crossing Imaginary Roots for Generic Quasipolynomials: A Vandermonde-Based Approach. IEEE Transactions on Automatic Control, 2016, 61 (6), pp.1601-1606. 10.1109/TAC.2015.2480175 . hal-01425757

\section{HAL Id: hal-01425757}

https://hal-centralesupelec.archives-ouvertes.fr/hal-01425757

Submitted on 7 Jul 2020

HAL is a multi-disciplinary open access archive for the deposit and dissemination of scientific research documents, whether they are published or not. The documents may come from teaching and research institutions in France or abroad, or from public or private research centers.
L'archive ouverte pluridisciplinaire HAL, est destinée au dépôt et à la diffusion de documents scientifiques de niveau recherche, publiés ou non, émanant des établissements d'enseignement et de recherche français ou étrangers, des laboratoires publics ou privés. 


\title{
Tracking the Algebraic Multiplicity of Crossing Imaginary Roots for Generic Quasipolynomials: A Vandermonde-Based Approach
}

\author{
Islam Boussaada $^{1}$ and Silviu-Iulian Niculescu ${ }^{2}$
}

\begin{abstract}
A standard way in analyzing dynamical systems consists in identifying and understanding the eigenvalues bifurcations when crossing the imaginary axis. Efficient methods for crossing imaginary roots identification exist. However, to the best of the authors knowledge, the multiplicity of such roots was not deeply investigated. In recent papers by the authors [1], [2], it is emphasized that the multiplicity of the zero spectral value can exceed the number of the coupled scalar delaydifferential equations and then provide a constructive approach Vandermonde-based allowing to an adaptive bound for such a multiplicity. Among others, it is shown that the zero spectral value multiplicity depends on the system structure (number of delays and number of non zero coefficients of the associated quasipolynomial) rather than the degree of the associated quasipolynomial [3]. This paper extends such a constructive approach in investigating the multiplicity of crossing imaginary roots $j \omega$ where $\omega \neq 0$ and establishes a link with a new class of functional confluent Vandermonde matrices. As a byproduct of the proposed approach, a symbolic algorithm for computing the LU-factorization for such matrices is provided. This allows computing a bound which is sharper than Polya-Szegö generic bound arising from the principle argument.
\end{abstract}

\section{INTRODUCTION}

Consider the following class of time-delay systems:

$$
\dot{x}=\sum_{k=0}^{N} A_{k} x\left(t-\tau_{k}\right)
$$

where $x=\left(x_{1}, \ldots, x_{n}\right) \in \mathbb{R}^{n}$ denotes the state-vector, under appropriate initial conditions belonging to the Banach space of continuous functions $\mathcal{C}\left(\left[-\tau_{N}, 0\right], \mathbb{R}^{n}\right)$. Here $\tau_{j}$, $j=1 \ldots N$ are strictly increasing positive constant delays, $\tau_{0}=0, \tau=\left(\tau_{1}, \ldots, \tau_{N}\right)$ and the matrices $A_{j} \in \mathcal{M}_{n}(\mathbb{R})$ for $j=0 \ldots N$. It is well known that the asymptotic behavior of the solutions of (1) is determined from its spectra $\aleph$ designating the set of the roots of the associated characteristic function denoted in the sequel $\Delta(z, \tau)$, often called quasipolynomial, that is a transcendental polynomial in the Laplace variable $z$ in which appears exponential terms induced by delays, see, [4] and [5]. The study of zeros of such a class of entire function [7] plays a crucial role especially in the analysis of asymptotic stability of the zero solution associated with System (1). Indeed, the zero solution is asymptotically stable if all the spectral values of (1) are in the open left-half complex plane [8]. Accordingly to this observation, the parameter space which is spanned by the coefficients of the polynomials $P_{i}$, can be split into stability and instability domains (Nothing else that the socalled D-decomposition, see for instance [8] and references therein). These two domains are separated by a boundary corresponding to the spectra consisting in roots with zero real parts called in the sequel Crossing Imaginary Roots (CIR). Identification of CIR for Time-delay systems may be done for instance by Rekasius Substitution [9] or Matrix Pencil [8] to mention only few efficient approaches. However, to the best of the authors knowledge, the multiplicity of such roots was not deeply investigated, even, the computation of the upper bound of the algebraic multiplicity of CIR still unsolved. The characterization of CIR i.e. identification of CIR as well as their associated multiplicities are useful to overcome several issues related to the qualitative description of solution of a given dynamical system with or without delays. Furthermore, the delays for which the characteristic equation has CIR are referred to as critical delays and the sensitivity of a given CIR, i.e., the derivative with respect to the delay, produce a complete stability picture by keeping track of the number of roots entering and leaving the right half-plane, see for instance [8] and [10]. Moreover, characterizing CIR has a twofold interest: in linear analysis as well as in nonlinear analysis perspectives. From a linear viewpoint, for instance, a complete characterization of CIR allows to estimate the number of the spectral values with positive real part (elements of $\aleph_{+}=\{z \in \mathbb{C}, \Delta(z, \tau)=0, \Re(z)>0\}$ ) for a given Time-delay system (1). Indeed, the main theorem from [11] emphasizes the link between $\downarrow\left(\aleph_{+}\right)$and $\aleph_{0}$ (the set of all CIR), both counted by multiplicity. Taking into account the quoted result and its potential consequences on the design of a new approach on the linear stability analysis of timedelay systems, a greater importance to the study of CIR become obvious. Such an issue is also emphasized from a nonlinear analysis viewpoint, which gives another motivation for the present investigation. When the unstable spectra is an empty set or equivalently the spectra is $\aleph=\aleph_{-} \cup \aleph_{0}$ (elements of $\aleph_{-}=\{z \in \mathbb{C}, \Delta(z, \tau)=0, \Re(z)<0\}$ ), a complete knowledge of CIR as well as their multiplicities becomes crucial predominatingly when the center manifold and the normal forms theory are involved for giving an accurate local qualitative description of the studied dynamical system.

A stimulating idea from [12], which consists in exploiting the properties of Vandermonde matrices to control one chain of integrators by delay blocks, suggest to the authors in [1] and [2] to extend the investigation of such structured matrices. A complete characterization of the root at the origin can be found in [1], [2]. It is shown that the multiplicity of the the root at the origin for a generic system is bounded by the degree of the associated quasipolynomial, a bound 
which was already depicted in [13]. ${ }^{1}$ Furthermore, in the sparse case, a sharper bound is established in [1] showing that the optimal multiplicity is related to the number of the non vanishing parameters rather than the degree of the quasipolynomial. The present work is a natural continuation of the last cited papers, its aim is three fold. First, it provides a symbolic algorithm for the LU-decomposition for a class of functional confluent Vandermonde matrices with trigonometric inputs (section III). Secondly, it extends the Vandermonde constructive approach established in [1] to characterize CIR on beyond the zero frequency (sections IV and V). Finally, in section V the bound of the algebraic multiplicity for a given CIR is provided.

\section{PREREQUisites}

\section{A. Problem formulation and used notations}

A given Time-delay system (1) is characterized by a quasipolynomial function $\Delta: \mathbb{C} \times \mathbb{R}^{N}+\rightarrow \mathbb{C}$ of the form:

$$
\Delta(z, \tau)=\operatorname{det}\left(z I-A_{0}-\sum_{k=1}^{N} A_{k} e^{-\tau_{k} z}\right)
$$

which gives,

$$
\begin{aligned}
\Delta(z, \tau) & =P_{0}(z)+\sum_{M^{k} \in S_{N, n}} P_{M^{k}}(z) e^{\sigma_{M^{k}} z} \\
& =P_{0}(z)+\sum_{k=1}^{\tilde{N}_{N, n}} P_{M^{k}}(z) e^{\sigma_{k} z}
\end{aligned}
$$

where $\sigma_{M^{k}}=-M^{k} \tau^{T}, \tau=\left(\tau_{1}, \ldots, \tau_{N}\right)$ is the delays vector, $S_{N, n}$ is the set of all the possible row vectors $M^{k}=$ $\left(M_{1}^{k}, \ldots, M_{N}^{k}\right)$ belonging to $\mathbb{R}^{N^{*}}$ such that $1 \leq M_{1}^{k}+\ldots+$ $M_{N}^{k} \leq n$ and $\tilde{N}_{N, n}=\natural\left(S_{N, n}\right)$. For instance,

$$
\begin{aligned}
S_{3,2}= & \{(1,0,0),(0,1,0),(0,0,1),(2,0,0),(1,1,0), \\
& (1,0,1),(0,2,0),(0,1,1),(0,0,2)\},
\end{aligned}
$$

is ordered first by increasing sums $\left(\sum_{i=1}^{N} M_{i}^{k}\right)$ then by lexicographical order. For instance, for $S_{3,2}$, one has $M^{2}=$ $(0,1,0), \tilde{N}_{3,2}=9$ and the cross-talk delay $-\sigma_{5}=\tau_{1}+\tau_{2}$, see [14]. It is also assumed that $a_{i, j}$ stands for the coefficient of the monomial $z^{j}$ in $P_{i}, a_{0}=\left(a_{0,0}, \ldots, a_{0, n}\right)^{\top}$ is the vector composed from the coefficients of the polynomial $P_{0}$ and $p=\left(a_{1,0}, \ldots, a_{1, n_{1}-1}, \ldots, a_{\tilde{N}, 0}, \ldots, a_{\tilde{N}, n_{\tilde{N}}-1}\right)^{\top}$.

Without any lost of generality and for the sake of simplicity, the quasipolynomial function can be written

$$
\Delta(z, \tau)=P_{0}(z)+\sum_{k=1}^{\tilde{N}} P_{k}(z) e^{\sigma_{k} z}
$$

where all the $\sigma_{k}$ can be assumed to be distinct and they can be considered as auxiliary delays for the quasipolynomial. Indeed, when for some value of the delay vector $\tau$ there exists some $k \neq k^{\prime}$ such that $M^{k} \tau^{T}=M^{k^{\prime}} \tau^{T}$ then the number of the polynomials is reduced by considering a new

\footnotetext{
${ }^{1}$ The degree of a given quasipolynomial is defined by the sum of the number of its polynomials plus their respective degrees minus one.
}

family of polynomials $\tilde{P}$ such that $\tilde{P}_{M^{k}}=P_{M^{k}}+P_{M^{k^{\prime}}}$. It follows from (2) that $P_{0}$ is a monic polynomial of degree $n$ in $z$ and the polynomials $P_{M^{k}}$ are such that $\operatorname{deg}\left(P_{M^{k}}\right)=$ $n-\sum_{s=1}^{N} M_{s}^{k} \leq(n-1), \forall M^{k} \in S_{N, n}$.

A given complex number $z_{0}$ is said to be a spectral value for (1) if $\Delta\left(z_{0}, \tau^{*}\right)=0$ for some delay vector $\tau^{*}$. Let denote by $\partial_{z}^{k} \Delta(z, \tau)$ the $k$-th derivative of $\Delta(z, \tau)$ given by (3) with respect to the variable $z$. A spectral value $z_{0}$ is of algebraic multiplicity $m \geq 1$ if $\Delta\left(z_{0}, \tau^{*}\right)=\partial_{z} \Delta\left(z_{0}, \tau^{*}\right)=$ $\ldots=\partial_{z}^{m-1} \Delta\left(z_{0}, \tau^{*}\right)=0$ and $\partial_{z}^{m} \Delta\left(z_{0}, \tau^{*}\right) \neq 0$. A generic bound for the multiplicity of a given spectral value is deduced from a complex analysis result by Pólya and Szegö in [13, p. 144]. Their proof is mainly based on Rouché Theorem and the principal argument. It gives a bound of the number of quasipolynomial roots in any horizontal strip $\beta \leq \operatorname{Im}(z) \leq \alpha$. Setting $\alpha=\beta=\omega$ gives the number of spectral values $z$ with $\operatorname{Im}(z)=\omega$, which is a natural bound for the multiplicity of a given CIR $z=j \omega$, denoted in the sequel $\natural_{P S}$. See also [15] for a modern formulation of the mentioned result.

\section{B. Integrator + two delay blocks:}

For the general linear scalar time-delay systems of arbitrary order with two delays, the geometric criteria from [16] allows to identify all CIR. However, the multiplicity of such roots was not explored.

For each pair of positive and distinct delays $\tau_{1}, \tau_{2}$ the equation $\dot{x}(t)+a_{1} x\left(t-\tau_{1}\right)+a_{2} x\left(t-\tau_{2}\right)=0$ admits a triple spectral value at $z_{0}=-\left(\tau_{1}+\tau_{2}\right) /\left(\tau_{1} \tau_{2}\right)$ if and only if $a_{1}=\tau_{2} \mathrm{e}^{-\tau_{1} z_{0}} /\left(\tau_{2} \tau_{1}-\tau_{1}^{2}\right), a_{2}=\tau_{1} \mathrm{e}^{-\tau_{2} z_{0}} /\left(\tau_{2} \tau_{1}-\tau_{2}^{2}\right)$. Moreover, under this configuration, the zero solution is asymptotically stable. However, the optimal multiplicity of a CIR is two and it is reached if and only if $\omega_{0}=$ $\pm \tan \left(\alpha_{0}\right) / \tau_{1}$ and $\tau_{2}=-\tau_{1} \alpha_{0} / \tan \left(\alpha_{0}\right)$

$$
a_{1}=\frac{\omega_{0} \cos \left(\omega_{0} \tau_{2}\right)}{\sin \left(\omega_{0}\left(\tau_{1}-\tau_{2}\right)\right)}, a_{2}=\frac{\omega_{0} \cos \left(\omega_{0} \tau_{1}\right)}{\sin \left(\omega_{0}\left(\tau_{2}-\tau_{1}\right)\right)},
$$

where $\alpha_{0}$ is a fixed point of $\alpha \mapsto \tan \circ \tan (\alpha)$ or equivalently, the root of $\alpha=\tan \circ \tan (\alpha)$ which is a nonempty set. Now, let us show the above claim. We start by the configuration allowing to a spectral value $z_{0}$ with multiplicity three, which is characterized by the solutions of the system of equations $\Delta\left(z_{0}, \tau\right)=\ldots=\partial_{z, \ldots, z}^{k} \Delta\left(z_{0}, \tau\right)=0$ for $k \in$ $\{0,1,2\}$ and $\partial_{z, \ldots, z}^{3} \Delta\left(z_{0}, \tau\right) \neq 0$ where the characteristic function is $\Delta(z) \stackrel{=}{=} z+a_{1} e^{-z \tau_{1}}+a_{2} e^{-z \tau_{2}}$, which can be also written

$\Delta(z)=\left(z-z_{0}\right)\left(1+\frac{\frac{\tau_{2} \mathrm{e}^{-\left(z-z_{0}\right) \tau_{1}}}{\tau_{1}\left(\tau_{2}-\tau_{1}\right)}+\frac{\tau_{1} \mathrm{e}^{-\left(z-z_{0}\right) \tau_{2}}}{\tau_{2}\left(\tau_{1}-\tau_{2}\right)}+z_{0}}{\left(z-z_{0}\right)}\right)$.

To prove the asymptotic stability of the zero solution, let assume that there exists $z_{1}=\zeta+j \eta$ a root of $\Delta(z)=0$ such that $\zeta \geq 0$. Thus, $z_{1}$ is a root of the second factor of $\Delta$, which gives:

$$
1=\sum_{i=1}^{2} \frac{c_{i} \mathrm{e}^{-\left(z_{1}-z_{0}\right) \tau_{i}}-1}{\tau_{i}\left(z_{1}-z_{0}\right)}, \text { where } c_{i}=\prod_{\substack{l=1 \\ l \neq i}}^{2} \frac{\tau_{l}}{\left(\tau_{l}-\tau_{i}\right)}
$$




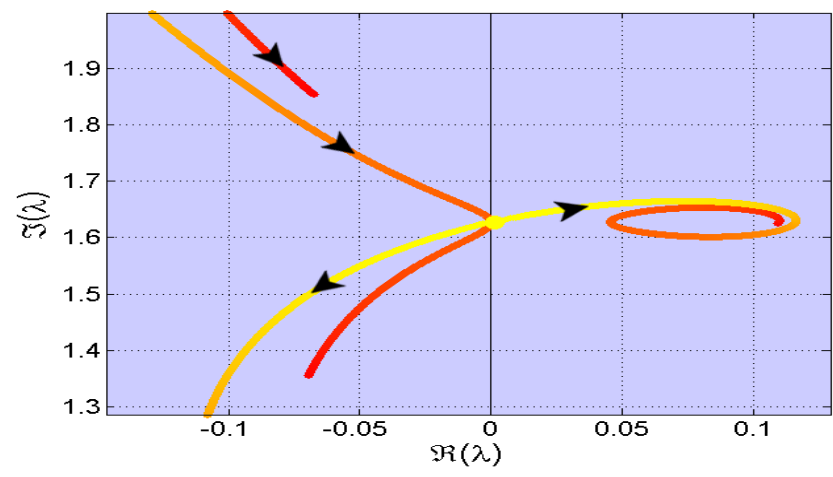

Fig. 1. Bifurcation of the double imaginary crossing root defined by (5) up to the standard normalisation $\tau_{2}^{*}=1$, the crossing frequency $\omega_{0}=$ 11.53849769 and the delay $\tau_{1}^{*}=0.1436275981$ is subject to a positive perturbation $(\epsilon=0 \ldots 6)$. The evolution of the spectral value from the nominal value $\epsilon=0$ (yellow) to the final value $\epsilon=6$ (red)

Hence, $1=\sum_{i=1}^{2} c_{i} \int_{0}^{1} \cos \left(\eta \tau_{i} t\right) \mathrm{e}^{-\left(\zeta-z_{0}\right) \tau_{i} t} d t$. Without any lost of generality, one assumes that $\tau_{1}<\tau_{2}$ which implies: $1 \leq \int_{0}^{1} \mathrm{e}^{-\left(\zeta-z_{0}\right) \tau_{1} t} d t$, proving the inconsistency of the hypothesis $\zeta>0$.

Let us check now that the upper bound of the multiplicity for CIR is two. Such a multiplicity is reached for $j \omega_{0}$ if and only if $\Delta\left(j \omega_{0}, \tau\right)=\partial_{z} \Delta\left(j \omega_{0}, \tau\right)=0$. Let us proceed by elimination. The first equation $\Delta\left(j \omega_{0}, \tau\right)=0$ gives, $a_{1} \cos \left(\omega_{0} \tau_{1}\right)+a_{2} \cos \left(\omega_{0} \tau_{2}\right)+$ $\left(\omega_{0}-a_{1} \sin \left(\omega_{0} \tau_{1}\right)-a_{2} \sin \left(\omega_{0} \tau_{2}\right)\right) j=0$. The vanishing of the real part allows to $a_{2}=-a_{1} \cos \left(\omega_{0} \tau_{1}\right) / \cos \left(\omega_{0} \tau_{2}\right)$, which is substituted in the imaginary part. After equating the resulting expression one has $a_{1}=$ $\omega \cos \left(\omega \tau_{2}\right) / \sin \left(\omega \tau_{1}-\omega \tau_{2}\right)$. The obtained value for $a_{1}$ is then itself substituted in $a_{2}$. The final values of $a_{1}$ and $a_{2}$ are then substituted in the equation $\partial_{z} \Delta(j \omega, \tau)=0$, which provides two equations (real and imaginary terms) in three left free parameters $\omega, \tau_{1}$ and $\tau_{2}$. Allowing as expected, to the expression of the crossing frequency $\omega_{0}$ as well as $\tau_{2}^{*}$ ( $\tau_{1}$ still being free).

Remark. For a given quasipolynomial function, the multiplicity of real roots may reach Polya-Szegö bound, see [2], [1]. However, the multiplicity of an imaginary crossing root may exceed the dimension of the delay-free system but never reach Polya-Szegö bound. A generalization of the above observation and further analysis illustrate the main result.

\section{Rekasius Substitution for a second order system}

It is well known that Rekasius substitution is a way for identifying the crossing roots for a given quasipolynomial, [14]. It consists in offsetting the exponential type transcendental terms in a given quasypolynomial function by the following rational function: $e^{-z \tau}=$ $(1-T z) /(1+T z)$, where $T$ is some positive real number. This transformation is accompanied with the relation: $\tau=$ $\frac{2}{\omega_{c}}\left(\arctan \left(\omega_{c} T\right)+k \pi\right), \quad k=0,1, \cdots$ where $\omega_{c} \in \mathbb{R}_{+}^{*}$ and $\omega_{c}$ denotes the crossing frequency. Il is worthy to note that such a relation associate to each solution of the transformed characteristic function in the auxiliary variable $T$ to a $2 \pi / \omega_{c}$-periodic countably infinite set of solutions of the original characteristic quasipolynomial function in the variable $\tau$, see for instance [9], [17]. However, the question on roots multiplicity-conservation by such a transformation may be more deeply investigated. For instance, by the following example, we show that even for the delay $\operatorname{margin}^{2} \tau^{0}$, such a multiplicity is not preserved by Rekasius substitution. Let consider the quasipolynomial function :

$$
f(z, \tau)=a_{0,0}+a_{0,1} z+z^{2}+\left(a_{1,0}+a_{1,1} z\right) \mathrm{e}^{-\tau z}
$$

with $a_{0,0}=\frac{\pi^{2}+4}{\pi^{2}-4}, a_{0,1}=a_{1,0}=\frac{-4 \pi}{\pi^{2}-4}, a_{1,1}=\frac{-8}{\pi^{2}-4}$.

First, one knows that the imaginary roots of $f$ are the ones of the following rational function in the variables $z$ and $T$ :

$$
\begin{aligned}
\tilde{f}(z, T)= & \frac{\mathcal{P}(z, T)}{\mathcal{Q}(z, T)} \text { where } \mathcal{Q}(z, T)=1+T_{1} z \text { and } \\
\mathcal{P}(z, T)= & \left(a_{0,0} z+a_{0,1} z^{2}+z^{3}-a_{1,0} z-a_{1,1} z^{2}\right) T \\
& +a_{0,0}+a_{0,1} z+z^{2}+a_{1,0}+a_{1,1} z .
\end{aligned}
$$

Elementary algebraic manipulations yields to $z_{0}=j$ is a root of $\tilde{f}$ when $T^{*}=1$. Thus, $z_{0}=j$ is a root of $f$ where the associated delays $\tau^{0}=\pi / 2$ is easily deduced. Finally, we check that when $\tau=\tau^{0}$, one has $z_{0}=j$ is a double root for $f$. However, the evaluation of $\partial_{z} \tilde{f}$ at $(z, T)=\left(z_{0}, T^{*}\right)$ gives: $\partial_{z} \tilde{f}\left(z_{0}, T^{*}\right)=2(\pi j-2) /(\pi+2)$. Thus, $z_{0}=j$ is a simple root for $\tilde{f}$ when $T=T^{*}$, leading us to the following:

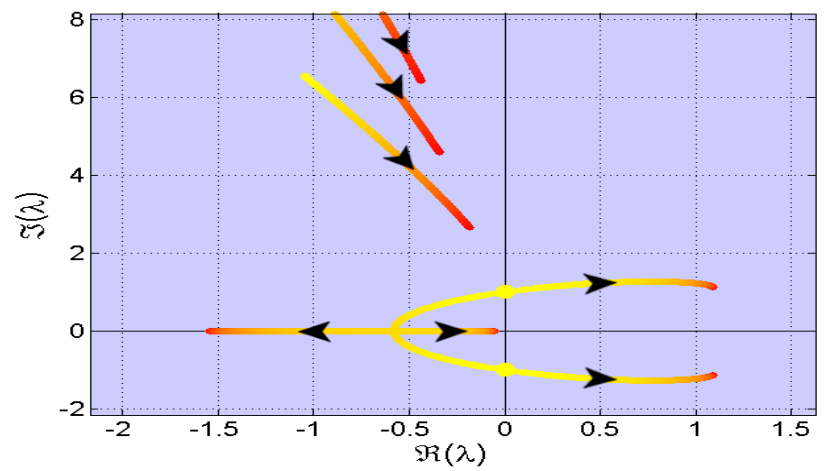

Fig. 2. Bifurcation of the double imaginary crossing root of (6) where the delay $\tau$ is subject to a positive perturbation $(\epsilon=0 \ldots 6)$. The evolution of the spectral value from the nominal value $\epsilon=0$ (yellow) to the final value $\epsilon=6$ (red).

Remark. For a given quasipolynomial function, Rekasius substitution yields to identify all roots on the imaginary axis but does not necessarily preserve their multiplicity. Furthermore, the other limitations of such a substitution arise when at least one of the polynomials (involved in the considered quasipolynomial) depend on $z$ and $\tau$. Which is obviously the case when exploring the multiplicity of CIR.

\footnotetext{
${ }^{2}$ Rekasius substitution asserts that when an imaginary root $j \omega_{0}$ is associated to a given delay $\tau^{0}$ then it is also associated to each value of the delay among the family $\left\{\tau^{k}\right\}_{k \in \mathbb{Z}}=\left\{\tau^{0}+k \frac{2 \pi}{\left|\omega_{0}\right|}\right\}_{k \in \mathbb{Z}}$. But, as it was emphasized in [10], if $j \omega_{0}$ is double crossing root associated with the delay $\tau=\tau^{0}$ then, $j \omega_{0}$ is a simple crossing root for any $\tau_{k}$.
} 


\section{Functional CONFLUENT VANDERMONDE MATRICES}

By functional confluent Vandermonde matrix $\Phi$ we designate the matrix defined by:

$$
\left\{\begin{aligned}
\Phi & =\left[\Phi_{1} \Phi_{2} \ldots \Phi_{M}\right] \in \mathcal{M}_{l, \delta}(\mathbb{R}), \\
\Phi_{i} & =\left[f\left(\sigma_{i}\right) f^{(1)}\left(\sigma_{i}\right) \ldots f^{\left(d_{i}-1\right)}\left(\sigma_{i}\right)\right], \\
f\left(\sigma_{i}\right) & =g\left(\sigma_{i}\right) \cdot\left[1 \ldots \sigma_{i}^{l-1}\right]^{T}, \quad \text { for } \quad 1 \leq i \leq M,
\end{aligned}\right.
$$

for sufficiently regular function $g \in \mathcal{C}^{k}(\mathbb{R})$. In the sequel, we are particularly interested by square matrices then it is assumed that $\sum_{i=1}^{M} d_{i}=\delta=l$. When $g(x)=1$ then we are dealing with the so-called confluent Vandermonde matrix. When additionally $d_{i}=1$ for $i=1 \ldots N$ then we recover the classical Vandermonde matrix and in this case $M=\delta$ since $\Phi$ is assumed to be a square matrix. An explicit recursive formula for the LU-factorization for a class of functional confluent Vandermonde matrices characterized by $g(x)=x^{k}$ is available in [1] or in its expended version [3]. The following notations are adopted. Let $\xi$ stands for the vector composed from $\sigma_{i}$ counting their repetition $d_{i}$ through columns of a given matrix $\Phi$, i.e. $\xi=(\underbrace{\sigma_{1}, \ldots, \sigma_{1}}_{d_{1}}, \ldots, \underbrace{\sigma_{M}, \ldots, \sigma_{M}}_{d_{M}})$.

It is important to note that the vector $\xi$, which will be called in the sequel the incidence vector, is a compact way to indicate the so-called incidence matrix associated with $\Phi$, see for instance [18]. It characterizes a given square functional confluent matrix $\Phi$ defined in (7) by indicating its variables as well as their repetitions which indicate the associated orders of derivations through its columns.

In the light of the above notations and under the setting $d_{0}=0$, without any loss of generality: $\xi_{k}=\xi_{d_{0}+\ldots+d_{r}+\alpha}=$ $\xi_{\sum_{l=0}^{\nu(k)-1} d_{l}+\varkappa(k)}$, where $0 \leq r \leq \nu-1$ and $\alpha \leq d_{r+1}$, here $\nu(k)$ denotes the index of component of $\sigma$ associated with $\xi_{k}$, that is $\sigma_{\nu(k)}=\xi_{k}$ and by $\varkappa(k)$ the order of $\xi_{k}$ in the sequence of $\xi$ composed only by $\sigma_{\nu(k)}$. Obviously, $\nu(k)=r+1$ and $\varkappa(k)=\alpha$.

Proposition 1. The functional confluent Vandermonde square matrix $\Phi$ given in (7) admits the following $L U$ factorization with unitary diagonal elements $L_{i, i}=1$ :

$$
\left\{\begin{aligned}
L_{i, 1}= & \sigma_{1}^{i-1} \quad \text { for } \quad 1 \leq i \leq \delta, \\
U_{1, j}= & \Phi_{1, j} \quad \text { for } \quad 1 \leq j \leq \delta, \\
L_{i, j}= & L_{i-1, j-1}+L_{i-1, j} \xi_{j} \quad \text { for } \quad 2 \leq j \leq i, \\
U_{i, j}= & (\varkappa(j)-1) U_{i-1, j-1}+U_{i-1, j}\left(\sigma_{\nu(j)}-\xi_{i-1}\right) \\
& \text { for } 2 \leq i \leq j .
\end{aligned}\right.
$$

Proof: Here, we follow the same steps of the proof given in [1], namely, a 2-D recurrence, see also the extended version [3].

The determinant of the functional confluent Vandermonde matrix $\Phi$ follows directly from (8):

Corollary 1. The determinant of the functional confluent Vandermonde matrix $\Phi$ is given by $\operatorname{det}(\Phi)=\prod_{j=1}^{\varrho}\left(U_{j, j}\right)$ where $U_{j, j}$ for $1 \leq j \leq \delta$ are defined by:

$$
\left\{\begin{array}{l}
U_{1,1}=g\left(\sigma_{1}\right), \\
U_{j, j}=g\left(\sigma_{k+1}\right) \prod_{l=1}^{k}\left(\sigma_{k+1}-\sigma_{l}\right)^{d_{l}} \\
\text { when } j=1+d_{k} \text { for } 1 \leq k \leq \tilde{N}-1, \\
U_{j, j}=\left(j-1-d_{k}\right) U_{j-1, j-1} \\
\text { when } d_{k}+1<j \leq d_{k+1} \text { for } 1 \leq k \leq \tilde{N}-1,
\end{array}\right.
$$

\section{Characterizing the Multiplicity of CIR:} COMPUTATIONAL ISSUES

Let denote by $c_{k}=\cos \left(\sigma_{k} \omega\right)$ and $s_{k}=\sin \left(\sigma_{k} \omega\right)$ and for a given real positif number $x,\lfloor x\rfloor$ designate the integer part of $x$ or equivalently the floor function at $x$.

Lemma 1. An imaginary complex number $z=j \omega$ is a root of $\partial_{z^{k}}^{k} \Delta(z, \tau)=0$ for $k \geq 0$ if and only if the coefficients of $P_{i}$ for $0 \leq i \leq \tilde{N}$ satisfy

$$
\alpha_{k}+\tilde{\alpha}_{k}=0 \text { and } \beta_{k}+\tilde{\beta}_{k}=0
$$

where

$$
\left\{\begin{aligned}
\alpha_{0}= & \sum_{\substack{i=0, i \text { even }}}^{n}(-1)^{\left\lfloor\frac{i}{2}\right\rfloor} \omega^{i} a_{0, i} \text { and } \beta_{0}=\sum_{\substack{i=1, i \text { odd }}}^{n}(-1)^{\left\lfloor\frac{i}{2}\right\rfloor} \omega^{i} a_{0, i}, \\
\alpha_{k}= & \partial_{\omega} \beta_{k-1} \text { and } \beta_{k}=\partial_{\omega} \alpha_{k-1} \forall k \geq 1, \\
\tilde{\alpha}_{k}= & \sum_{i=1}^{\tilde{N}} \sum_{l=0}^{d_{i}} a_{i, l} \frac{\partial^{l}\left(c_{i} \sigma_{i}{ }^{k}\right)}{\partial \sigma_{i}^{l}} \text { and } \tilde{\beta}_{k}=\sum_{i=1}^{\tilde{N}} \sum_{l=0}^{d_{i}} a_{i, l} \frac{\partial^{l}\left(s_{i} \sigma_{i}{ }^{k}\right)}{\partial \sigma_{i}^{l}} .
\end{aligned}\right.
$$

Let denote by $\gamma_{k}=\left(\alpha_{k}+\tilde{\alpha}_{k}\right)^{2}+\left(\beta_{k}+\tilde{\beta}_{k}\right)^{2}$ and the variety As a direct consequence of the above Lemma 1, one has:

Proposition 2. An imaginary crossing root $z=j \omega$ for $E q$ (3) is of multiplicity $m$ if and only if the variety $\mathcal{V}_{m}$ is non empty and $\gamma_{m+1} \neq 0$, where

$$
\begin{aligned}
\mathcal{V}_{m}= & \left\{\left(\omega, a_{0,0}, \ldots, a_{\tilde{N}, d_{\tilde{N}}}, \sigma_{1}, \ldots, \sigma_{\tilde{N}}\right) \in \mathbb{R}^{n+\delta+1} \times \mathbb{R}_{+}^{\tilde{N}},\right. \\
& \left.\gamma_{k}=0 \text { for } k=0, \ldots, m-1\right\}
\end{aligned}
$$

Remark. The above proposition provide a system of trigonometric-polynomials to solve. This allows to characterize multiple CIR. A standard approche in solving such systems of nonlinear trigonometric polynomials suggest to consider the $\sin \left(\omega \sigma_{i}\right)$ and $\cos \left(\omega \sigma_{i}\right)$ functions as new variables $s_{i}$ and $c_{i}$ respectively and then intentionally add some equations expressing $s_{i}^{2}+c_{i}^{2}=1$. Thus, Groebner basis approches may be involved to characterize the solutions of the problem, [19]. Unfortunately, in our case, even though the added algebraic equations the links between the variables $s_{i}$ and $c_{i}$ are provided, but, the link with the variable $\omega$ (which is involved in the argument of cos and sin) may be lost. This often introduce inconsistant solutions.

In what follow, we provide a linear algebra framework allowing to overcome the emphasized computational issue. 


\section{LinKS With FUnCTIONAL VANDERMONDE MATRICES}

In the light of Lemma 1 as well as the auxiliary results established in section III, we are now able to prove the following proposition. For the sake of simplicity, for a given positive integer $m$ let $\alpha^{m}, \beta^{m}$ stand for the vectors:

$$
\left\{\begin{array}{l}
\alpha^{m}\left(a_{0}, \omega\right)=-\left(\alpha_{0}, \ldots, \alpha_{m-1}\right)^{\top}, \\
\beta^{m}\left(a_{0}, \omega\right)=-\left(\beta_{0}, \ldots, \beta_{m-1}\right)^{\top} .
\end{array}\right.
$$

Let $\varrho=\sum_{i=1}^{\tilde{N}}\left(d_{i}+1\right)$, we define the functional confluent matrices $A_{m}$ and $B_{m}$ belonging to $\mathcal{M}_{m, \varrho}(\mathbb{R})$ and characterized by the incidence vector $\xi=(\underbrace{\sigma_{1}, \ldots, \sigma_{1}}_{d_{1}}, \ldots, \underbrace{\sigma_{\tilde{N}}, \ldots, \sigma_{\tilde{N}}}_{d_{\tilde{N}}})$ as well as the respective functions $g_{A}(x)=\cos (\omega x)$ and $g_{B}(x)=\sin (\omega x)$.

Proposition 3. An imaginary crossing root $z=j \omega$ for $E q$ (3) is of multiplicity $m$ if and only if its frequency $\omega$ satisfy the following linear system:

$$
\left\{\begin{array}{l}
A_{m}(\omega, \sigma) \cdot p=\alpha^{m}\left(a_{0}, \omega\right) \\
B_{m}(\omega, \sigma) \cdot p=\beta^{m}\left(a_{0}, \omega\right) .
\end{array}\right.
$$

Proof: The proposition follows directly from Lemma 1 by considering the two ideals consisting (respectively) in the real part $\alpha_{k}+\tilde{\alpha}_{k}$ (respectively the imaginary part $\beta_{k}+\tilde{\beta}_{k}$ ) of the successive derivatives of the quasipolynomial function. Which gives rise to the system (11).

Corollary 2. When the square matrices $A_{\varrho}$ and $B_{\varrho}$ are non degenerate then the multiplicity of any imaginary crossing root $z=j \omega_{0}$ for $E q$ (3) is bounded by $\varrho$.

Corollary 3. The functional confluent Vandermonde matrix $A_{\varrho}$ (respectively $\left.B_{\varrho}\right)$ is invertible if and only if $\forall 1 \leq i \neq$ $j \leq \tilde{N}$ we have $\omega \sigma_{i} \neq \frac{\pi}{2}+k \pi$ and $\sigma_{i} \neq \sigma_{j}$ (respectively $\omega \sigma_{i} \neq k \pi$ and $\sigma_{i} \neq \sigma_{j}$ ).

Remark. A necessary and sufficient condition for $A_{\varrho}$ and $B_{\varrho}$ to be invertible is $\sigma_{i} \neq \sigma_{j} \forall 1 \leq i \neq j \leq \tilde{N}$ and $\omega \sigma_{i} \neq$ $\frac{k \pi}{2}, k \in \mathbb{N}$. Moreover, a given CIR has the multiplicity $\varrho$ if the both of the linear systems given by (11) admit the same real solution. Thus, the above multiplicity bound for a given CIR is sharper than $\sharp_{P S}$ Polya-Szegö bound. Indeed, $\sharp_{P S}=n+\sum_{i=1}^{\tilde{N}}\left(d_{i}+1\right)$.

\section{ILluStRATIVE EXAMPLES}

\section{A. Scalar equation with two delays}

Let us consider the scalar delay equation: $\dot{x}(t)+a_{1,0} x(t-$ $\left.\tau_{1}\right)+a_{0,1} x\left(t-\tau_{2}\right)+a_{0,0} x(t)=0$. Here $p=\left(a_{1,0}, a_{0,1}\right)^{\top}$ and $\tilde{N}=2$. Following Corollary 1 the optimal multiplicity of a given CIR is bounded by $\varrho=2<\sharp_{P S}=3$. Moreover, a necessary condition for a CIR to reach multiplicity two is $\tau_{1} \neq \tau_{2}$ and $\omega \tau_{i} \neq \frac{k \pi}{2}$. Indeed, in such a configuration, we have $\sigma_{1,0}=-\tau_{1}$ and $\sigma_{0,1}=-\tau_{2}$ and the functional
Vandermonde matrices:

$$
\begin{aligned}
A_{2} & =\left[\begin{array}{cc}
\cos \left(\sigma_{1,0} \omega\right) & \cos \left(\sigma_{0,1} \omega\right) \\
\sigma_{1,0} \cos \left(\sigma_{1,0} \omega\right) & \sigma_{0,1} \cos \left(\sigma_{0,1} \omega\right)
\end{array}\right], \\
B_{2} & =\left[\begin{array}{cc}
\sin \left(\sigma_{1,0} \omega\right) & \sin \left(\sigma_{0,1} \omega\right) \\
\sigma_{1,0} \sin \left(\sigma_{1,0} \omega\right) & \sigma_{0,1} \sin \left(\sigma_{0,1} \omega\right)
\end{array}\right], \\
\alpha^{2} & =\left[\begin{array}{c}
-a_{0,0} \\
-1
\end{array}\right] \text { and } \beta^{2}=\left[\begin{array}{c}
-\omega \\
0
\end{array}\right] .
\end{aligned}
$$

Using Proposition 1, one proceeds by LU-factorisation for each of the matrices given in System (11) and obtains a necessary and sufficient condition for a given CIR to reach its optimal multiplicity, that is to be double:

$$
\left\{\begin{array}{l}
\left(a_{0,0} \sigma_{0,1}-1\right) \sin \left(\omega \sigma_{1,0}\right)=\omega \sigma_{0,1} \cos \left(\omega \sigma_{1,0}\right) \\
\left(a_{0,0} \sigma_{1,0}-1\right) \sin \left(\omega \sigma_{0,1}\right)=\omega \sigma_{1,0} \cos \left(\omega \sigma_{0,1}\right)
\end{array}\right.
$$

When $a_{0,0}=0$ then one recovers example II-B.

\section{B. Planar system with two delays}

One associates to the generic planar system with two delays the characteristic quasipolynomial function $\Delta(z, \tau)=$ $P_{0}(z)+P_{1,0}(z) e^{-z \tau_{1}}+P_{0,1}(z) e^{-z \tau_{2}}+P_{2,0}(z) e^{-2 z \tau_{1}}+$ $P_{0,2}(z) e^{-2 z \tau_{2}}+P_{1,1}(z) e^{-z\left(\tau_{1}+\tau_{2}\right)}$. Furthermore, following Corollary 3 the associated functional Vandermonde matrices $A_{7}(\omega, \sigma)$ and $B_{7}(\omega, \sigma)$ are non singular when $\sigma_{i} \neq \sigma_{j}$ $\forall 1 \leq i \neq j \leq 7$ and $\omega \sigma_{i} \neq \frac{k \pi}{2}, k \in \mathbb{N}$. Under the assumption of Proposition 2, a given CIR has at most the multiplicity 7 . However, the Polya-Szegö bound is $\sharp_{P S}=9$.

\section{CONCLUSION}

In this paper we addressed the question on the characterization of multiple CIR for time-delay systems as well as their admissible and optimal multiplicities. The considered configuration is generic, allowing to the link with some Hermite interpolation problem through functional confluent Vandermonde matrices with trigonometric inputs. A general formulas for LU-factorization of such matrices is established. To the best of our knowledge, the use of this class of matrices as well as their factorisation could not be found in standard linear algebra textbooks. Finally, we emphasize that our approach may be immediately extended to characterize CIR for neutral systems.

\section{ACKNOWLEDGMENT}

\section{REFERENCES}

[1] I. Boussaada, S.-I. Niculescu, Computing the codimension of the singularity at the origin for delay systems: The missing link with birkhoff incidence matrices, 21st International Symposium on Mathematical Theory of Networks and Systems (2014) $1-6$.

[2] I. Boussaada, D. Irofti, S.-I. Niculescu, Computing the codimension of the singularity at the origin for time-delay systems in the regular case: A vandermonde-based approach, 13th European Control Conference (2014) $1-6$

[3] I. Boussaada, S.-I. Niculescu, On codimension of zero singularities for time-delay systems: A link with vandermonde and birkhoff incidence matrices, Preprint (2013) 1-30.

[4] O. Diekmann, S. V. Gils, S. V. Lunel, H. Walther, Delay equations, Vol. 110 of Applied Mathematical Sciences, Functional, complex, and nonlinear analysis, Springer-Verlag, New York, 1995. 
[5] R. Bellman, K. L. Cooke, Differential-difference equations, Academic Press, New York, 1963.

[6] L. V. Ahlfors, Complex Analysis, McGraw-Hill, Inc., 1979.

[7] B. J. Levin, R. P. Boas, Distribution of zeros of entire functions, Translations of Mathematical Monographs, American Mathematical Society, Providence, Rhode Island, 1964, trad. du russe : Raspredelenie kosnej celyh funkcij.

[8] W. Michiels, S.-I. Niculescu, Stability and stabilization of time-delay systems, Vol. 12 of Advances in Design and Control, Society for Industrial and Applied Mathematics (SIAM), Philadelphia, PA, 2007.

[9] N. Olgac, R. Sipahi, An exact method for the stability analysis of timedelayed lit systems, IEEE Trans. Automat. Contr. 47 (2002) 793-797.

[10] E. Jarlebring, W. Michiels, Invariance properties in the root sensitivity of time-delay systems with double imaginary roots, Automatica 46 (6) (2010) 1112 - 1115 .

[11] B. Hassard, Counting roots of the characteristic equation for linear delay-differential systems, Journal of Differential Equations 136 (2) (1997) $222-235$

[12] S.-I. Niculescu, W. Michiels, Stabilizing a chain of integrators using multiple delays, IEEE Trans. on Aut. Cont. 49 (5) (2004) 802-807.

[13] G. Polya, G. Szegö, Problems and Theorems in Analysis, Vol. I: Series, Integral Calculus, Theory of Functions, Springer-Verlag, New York, Heidelberg, and Berlin, 1972.

[14] R. Sipahi, N. Olgac, Complete stability robustness of third-order $\{$ LTI $\}$ multiple time-delay systems, Automatica 41 (8) (2005) 1413 - 1422

[15] F. Wielonsky, A Rolle's theorem for real exponential polynomials in the complex domain, J. Math. Pures Appl. 4 (2001) 389-408.

[16] K. Gu, S.-I. Niculescu, J. Chen, On stability crossing curves for general systems with two delays, Journal of Mathematical Analysis and Applications 311 (1) (2005) 231 - 253

[17] U. Munz, C. Ebenbauer, T. Haag, F. Allgower, Stability analysis of time-delay systems with incommensurate delays using positive polynomials, Automatic Control, IEEE Transactions on 54 (5) (2009) 1019-1024.

[18] L. Gonzalez-Vega, Applying quantifier elimination to the Birkhoff interpolation problem, J. Symbolic. Comp. 22 (1) (1996) 83-104.

[19] D. Cox, J. Little, D. O'Shea, Ideals, varieties, and algorithms. An introduction to computational algebraic geometry and commutative algebra, Undergraduate Texts in Mathematics, Springer, New York, 2007.

\section{APPENDIX}

Proof of Lemma 1 We emphasize that the coefficient $\alpha_{k}$ (respectively $\beta_{k}$ ) is the real part (respectively the imaginary part ) of the polynomial $P_{0}^{(k)}(j \omega)$. Furthermore, $\alpha_{k}$ and $\beta_{k}$ are multivariate polynomials in $\omega$ and in the components of $a_{0}$ (the coefficients of $P_{0}$ in $z$ ). It is also important to note that, $\alpha_{s+1}=\beta_{s+1}=0$ for all integer $s \geq$ $n$. Consider the quasipolynomial given in (4). A complex number $j \omega$ is a root of $\Delta(z, \tau)=0$ if and only if $P_{0}(j \omega)+$ $\sum_{k=1}^{\tilde{N}} P_{k}(j \omega) e^{j \sigma_{k} \omega}=0$ which gives:

$$
\begin{aligned}
& a_{0,0}+\ldots+a_{0, n}(j \omega)^{n}+\left(a_{1,0}+\ldots+a_{1, d_{1}}(j \omega)^{d_{1}}\right) e^{j \sigma_{1} \omega} \\
& +\ldots+\left(a_{\tilde{N}, 0}+\ldots+a_{\tilde{N}, d_{\tilde{N}}}(j \omega)^{d_{\tilde{N}}}\right) e^{j \sigma_{\tilde{N}} \omega}=0
\end{aligned}
$$

Using Euler formula, one obtains:

$$
\begin{aligned}
& \alpha_{0}+j \beta_{0}+\left(a_{1,0}+\ldots+a_{1, d_{1}}(j \omega)^{d_{1}}\right)\left(c_{1}+j s_{1}\right) \\
& +\ldots+\left(a_{\tilde{N}, 0}+\ldots+a_{\tilde{N}, d_{\tilde{N}}}(j \omega)^{d_{\tilde{N}}}\right)\left(c_{\tilde{N}}+j s_{\tilde{N}}\right)=0 .
\end{aligned}
$$

Now, extracting the real and imaginary parts of the above expression allows us to prove (9) for $k=0$ :

$$
\left\{\begin{array}{l}
\alpha_{0}+c_{1} a_{1,0}-\omega s_{1} a_{1,1}-\omega^{2} c_{1} a_{1,2}+\ldots+\omega^{d_{1}} \frac{\partial^{d_{1}} c_{1}}{\partial \sigma_{1}^{d_{1}}} a_{1, d_{1}} \\
+\ldots+c_{\tilde{N}} a_{\tilde{N}, 0}+\ldots+\omega^{d_{\tilde{N}}} \frac{\partial^{d_{\tilde{N}}} c_{\tilde{N}}}{\partial \sigma_{\tilde{N}}^{d_{\tilde{N}}}}=0, \\
\beta_{0}+s_{1} a_{1,0}+\omega c_{1} a_{1,1}-\omega^{2} s_{1} a_{1,2}+\ldots+\omega^{d_{1}} \frac{\partial^{d_{1}} s_{1}}{\partial \sigma_{1}^{d_{1}}} a_{1, d_{1}} \\
+\ldots+s_{\tilde{N}} a_{\tilde{N}, 0}+\ldots+\omega^{d_{\tilde{N}}} \frac{\partial^{d_{\tilde{N}}} s_{\tilde{N}}}{\partial \sigma_{\tilde{N}}^{d_{\tilde{N}}}}=0 .
\end{array}\right.
$$

Consider the first partial derivatives of the quasipolynomial:

$$
\begin{aligned}
& \partial_{z} \Delta(z, \tau)=P_{0}^{\prime}(z)+\sum_{i=1}^{\tilde{N}}\left(P_{i}^{\prime}(z)+\sigma_{i} P_{i}(z)\right) e^{\sigma_{i} z}, \\
& \partial_{z^{2}}^{2} \Delta(z, \tau)=P_{0}^{\prime \prime}(z)+\sum_{i=1}^{\tilde{N}}\left(P_{i}^{\prime \prime}(z)+2 \sigma_{i} P_{i}^{\prime}(z)+\sigma_{i}^{2} P_{i}(z)\right) e^{\sigma_{i} z} .
\end{aligned}
$$

By induction we arrive to :

$$
\partial_{z^{k}}^{k} \Delta(z, \tau)=P_{0}^{(k)}(z)+\sum_{i=1}^{\tilde{N}} e^{\sigma_{i} z} \sum_{m=0}^{k}\left(\begin{array}{c}
m \\
k
\end{array}\right) \sigma_{i}^{k-m} P_{i}^{(m)}(z) .
$$

Now, assume that $z=j \omega$ is a root of $\partial_{z_{k}}^{k} \Delta(z, \tau)=0$ for $k \geq 1$ and let us show that (9) is satisfied.

$$
0=P_{0}^{(k)}(j \omega)+\sum_{i=1}^{\tilde{N}} \sum_{m=0}^{k}\left(\begin{array}{c}
m \\
k
\end{array}\right) \sigma_{i}^{k-m} P_{i}^{(m)}(j \omega)\left(c_{i}+j s_{i}\right) .
$$

Thus, $\alpha_{k}=-\mathcal{R} e\left(\sum_{i=1}^{\tilde{N}} \sum_{m=0}^{k}\left(\begin{array}{c}m \\ k\end{array}\right) \sigma_{i}^{k-m} P_{i}^{(m)}(j \omega)\left(c_{i}+j s_{i}\right)\right)$. Obviously, when $k \geq n$ then $P_{0}^{(k)}(j \omega)=\alpha_{k}+j \beta_{k}=0$. The right hand side of the above equality is nothing else than $\tilde{\alpha}_{k}$. Indeed,

$$
\begin{aligned}
& \mathcal{R} e\left(\sum_{i=1}^{\tilde{N}} \sum_{m=0}^{k}\left(\begin{array}{c}
m \\
k
\end{array}\right) \sigma_{i}^{k-m} \sum_{l=0}^{d_{i}}\left[a_{i, l} z^{l}\right]_{z=j \omega}^{(m)}\left(c_{i}+j s_{i}\right)\right)= \\
& \mathcal{R} e\left(\sum_{i=1}^{\tilde{N}} \sum_{l=0}^{d_{i}} a_{i, l} \sum_{m=0}^{k}\left(\begin{array}{c}
m \\
k
\end{array}\right) \sigma_{i}^{k-m}\left[z^{l}\right]_{z=j \omega}^{(m)}\left(c_{i}+j s_{i}\right)\right)= \\
& \sum_{i=1}^{\tilde{N}} \sum_{l=0}^{d_{i}} a_{i, l} \mathcal{R} e\left(\sum_{m=0}^{k}\left(\begin{array}{c}
m \\
k
\end{array}\right) \sigma_{i}^{k-m}\left[z^{l}\right]_{z=j \omega}^{(m)}\left(c_{i}+j s_{i}\right)\right)=\tilde{\alpha}_{k}
\end{aligned}
$$

since one shows by induction w.r.t to $l$ (for a fixed $k$ ) that

$$
\frac{\partial^{l}\left(c_{i} \sigma_{i}^{k}\right)}{\partial \sigma_{i}^{l}}=\mathcal{R} e\left(\sum_{m=0}^{k}\left(\begin{array}{c}
m \\
k
\end{array}\right) \sigma_{i}^{k-m}\left[z^{l}\right]_{z=j \omega}^{(m)}\left(c_{i}+j s_{i}\right)\right) \text {. }
$$

